\title{
COLABORAÇÃO CLIENTE-FORNECEDOR NO DESENVOLVIMENTO DE UM SISTEMA PRODUTO-SERVIÇO PARA REDE DE TELECOMUNICAÇÃOO
}

Lílian Salgueiro Azevedo (liliansaazevedo@gmail.com) - Departamento de Engenharia de Produção, Universidade Federal de São Carlos.

José Carlos de Toledo (toledo@dep.ufscar.br) - Departamento de Engenharia de Produção, Universidade Federal de São Carlos.

\begin{abstract}
RESUMO
Este trabalho tem como objetivos analisar como os atores de uma empresa cliente de uma oferta de Sistema Produto-Serviço (SPS) interagem entre si e com outros atores, principalmente com os da empresa fornecedora, e identificar as melhorias que podem ser feitas na colaboração clientefornecedor. $O$ trabalho foi desenvolvido por meio do estudo de caso em uma empresa de telecomunicação francesa, a qual compra uma oferta de SPS fornecido por uma empresa de telecomunicação sueca. Foram realizadas entrevistas semiestruturadas para coleta de dados na empresa cliente. O mapa dos atores e o mapa do sistema foram elaborados durante as entrevistas a fim de identificar os atores envolvidos, os tipos de interações e o fluxo de informações entre eles. A conclusão é que empresa cliente precisa fazer melhorias internas, tais como aumentar a rapidez na tomada de decisão, melhorar seu fluxo de informações entre os atores e reforçar a interação entre os departamentos. A colaboração com a empresa fornecedora pode ser aperfeiçoada, como, por exemplo, realizando reuniões regulares que permitam trocar informações importantes sobre o desenvolvimento do SPS. Os resultados também trazem à tona os benefícios e os pontos-fracos quando se trabalha em um SPS.
\end{abstract}

Palavras-chave: Sistema produto-serviço, colaboração cliente-fornecedor, desenvolvimento de sistema produto-serviço

Área: Desenvolvimento de Sistemas Produto-Serviço (PSS)

\section{INTRODUÇÃO}

O Sistema Produto-Serviço (SPS) é considerado uma estratégia inovadora para as empresas e pode ser definido como uma combinação de produtos tangíveis e serviços intangíveis que em conjunto são capazes de atender às necessidades dos clientes finais (TUKKER; TISCHNER, 2006).

Os serviços por serem intangíveis são mais complexos quando se trata de medir a qualidade do resultado final. Isso exige mais das empresas que fornecem um SPS, para não só oferecer serviços que satisfaçam a demanda do cliente, mas também a sua experiência. A complexidade também torna mais difícil para o cliente fazer exigências sobre o serviço que vai lhe ser entregue (AHLSTROM, 2004).

As possibilidades de melhoria do SPS prestado pelo fornecedor são difíceis de serem identificadas pelo cliente, uma vez que o SPS pode envolver muitos atores. Portanto, com o aumento dos requisitos dos clientes e da concorrência, torna-se vantajoso para as empresas 
identificar o papel desses atores envolvidos em uma oferta SPS. Em muitos casos, para que seja possível a melhoria, os atores tanto do cliente quanto do fornecedor devem ter acesso a informações importantes, saber como essas informações são transferidas e ter uma ideia de quem usará as informações que eles possuem (LINDAHL; SAKAO; CARLSSON, 2014).

Quando uma empresa cliente compra uma oferta de SPS, ela e seu fornecedor começam a ter um contato mais próximo durante a fase de uso através da troca de conhecimento. Por consequência, o diálogo entre eles tende a aumentar, sendo uma importante fonte de informação, por exemplo, sobre como melhorar o desenvolvimento ou como personalizar o SPS, a fim de melhorar o valor recebido pelo cliente (LINDAHL; SAKAO; RÖNNBÄCK, 2009). Consequentemente, o fluxo de informação é fundamental para a realização eficaz de um SPS (SAKAO; RÖNNBÄCK; SANDSTRÖM, 2013). Para tornar a informação útil, é importante obter uma boa compreensão sobre os transmissores e receptores para que a informação seja usada de forma adequada (LINDAHL; SAKAO; CARLSSON, 2014).

Este trabalho tem como objetivo analisar como os atores de uma empresa cliente de uma oferta de SPS interagem entre si e com outros atores, principalmente com os da empresa fornecedora, e identificar melhorias que podem ser feitas neste sistema. Para isso, foi realizado estudo de caso em uma empresa francesa de telecomunicação que compra uma oferta de SPS.

\section{MÉTODO DE PESQUISA}

Para atingir o objetivo desta pesquisa, a maneira de entender a complexidade da colaboração e identificar as oportunidades de melhoria nas interações cliente-fornecedor foi entrevistar os próprios atores. A realização de entrevistas semiestruturadas individuais são utilizadas neste estudo, uma vez que permitem que os entrevistados expressem livremente sua perspectiva sobre a colaboração e identifiquem os atores envolvidos e suas conexões. A coleta de dados sobre o estado da arte atual na empresa participante foi realizada através de oito entrevistas semiestruturadas, sendo quatro face-a-face e quatro por videoconferência, entre elas com o Vice-presidente Global M2M, o Diretor de Marketing de Produto e o Diretor de Roaming M2M. As entrevistas tiveram duração entre 40-60 minutos e todas foram registradas por meio de um gravador e com autorização dos entrevistados.

A elaboração do roteiro de entrevista, seleção dos entrevistados, transcrição das entrevistas, análise, verificação e interpretação dos dados coletados, seguiram as etapas do processo de entrevista proposto por Brinkmann e Kvale (2015). Para validar o roteiro de entrevista foi realizada uma entrevista semiestruturada face-a-face com um trabalhador da empresa, o qual foi o primeiro contato que respondeu a solicitação da entrevista enviada por e-mail. Nesta entrevista, o entrevistado sugeriu quatro possíveis respondentes, os quais devido ao cargo que possuem na empresa e o envolvimento com a oferta SPS foram os primeiros a serem entrevistados. Assim, a seleção dos últimos quatro entrevistados seguiram a recomendação de Babbie (2013), o qual explica que em vez de decidir antecipadamente quem vai ser entrevistado, o pesquisador os seleciona gradualmente perguntando aos respondentes para sugerir quem será o próximo entrevistado. Todas as entrevistas foram transcritas uma vez que foram gravadas. De acordo com Brinkmann e Kvale (2015), após a transcrição, a análise de dados deve ser feita. Neste trabalho foram feitas as mesmas perguntas aos participantes a fim de poder comparar as respostas de cada um.

A forma mais comum de análise, e que foi utilizada neste trabalho, é a análise de conteúdo, que consiste na leitura detalhada de todos os dados transcritos, na identificação de palavras e grupos de palavras que fazem sentido para a pesquisa, bem como a classificação em 
categorias ou temas que têm semelhanças em um assunto particular (BRINKMANN; KVALE, 2015). Uma vez que grande parte dos dados coletados é sensível ou confidencial, foi decidido não descrever dados detalhados sobre os processos da empresa participante.

\section{ESTUDO DE CASO}

O trabalho foi realizado por meio do estudo de caso da empresa de telecomunicação francesa a fim de abordar o ponto de vista do cliente de uma oferta de SPS. A empresa cliente trabalha em parceria com outras empresas multinacionais do mesmo ramo de negócio. Para enriquecer seus produtos machine-to-machine (M2M) e portfólio de soluções a fim de atender principalmente as necessidades de roaming internacional de seus clientes, essas empresas parceiras optaram por trabalhar em colaboração com a empresa de telecomunicação sueca, a qual fornece uma oferta de SPS. Essa oferta é uma plataforma de conectividade em nuvem, fornecida como serviço, entre dispositivos M2M. Todas essas empresas (clientes e fornecedor) trabalham em colaboração no desenvolvimento e aprimoramento desta oferta de SPS. Essa plataforma é de propriedade do fornecedor, o qual fornece apenas o serviço aos clientes.

$\mathrm{O}$ foco deste estudo é a colaboração cliente-fornecedor entre a empresa francesa e a sueca. $\mathrm{O}$ desafio desta colaboração está, principalmente, no fato de que a oferta de SPS é uma oferta compartilhada e este novo modo de trabalho envolve muitos atores e implica em significativas mudanças na cultura das empresas e também nas relações entre os atores internos e os externos.

\subsection{Interação entre cliente e fornecedor}

Para analisar e compreender como os atores da empresa cliente interagem entre si e com outros atores, principalmente com os da empresa fornecedora, os mapas dos atores e do sistema (Actors Map and System Map) foram feitos baseados no método de mapeamento para Sistemas Produto-Serviço propostos por Lindahl, Sakao e Carlsson (2014).

Durante as entrevistas com a empresa cliente foi investigado e identificado quem eram os atores envolvidos e qual tipo de informação trocavam entre eles, tendo como resultado os mapas dos atores e do sistema, conforme constam nas figura 1 e 2, respectivamente.

Na figura 1 os atores são representados pelas caixas e as interações, pelas setas. Exemplos de atores da empresa cliente são: departamento de marketing, diretor de produto, gerente de projeto e departamento técnico. Exemplos de atores da empresa fornecedora são: departamento de vendas e gerente de projetos. O mapa também envolve atores que tradicionalmente podem ser considerados fora da oferta de SPS por exemplo, operadores de roaming e empresa de consultoria.

O objetivo do mapa dos atores é apoiar a identificação dos atores relevantes e suas interações e requisitos que podem ser importantes quando se desenvolve um SPS (LINDAHL; SAKAO; CARLSSON, 2014). O mapa dos atores do SPS também apoiou a identificação dos atores que recebem grandes quantidades de informações, existindo o risco desses atores serem sobrecarregados de informações as quais podem ser perdidas por não serem transferidas aos atores que precisam dela. 
Figura 1. Mapa dos atores do SPS que ilustra os atores participantes e suas conexões. (Mapa alterado devido a confidencialidade).

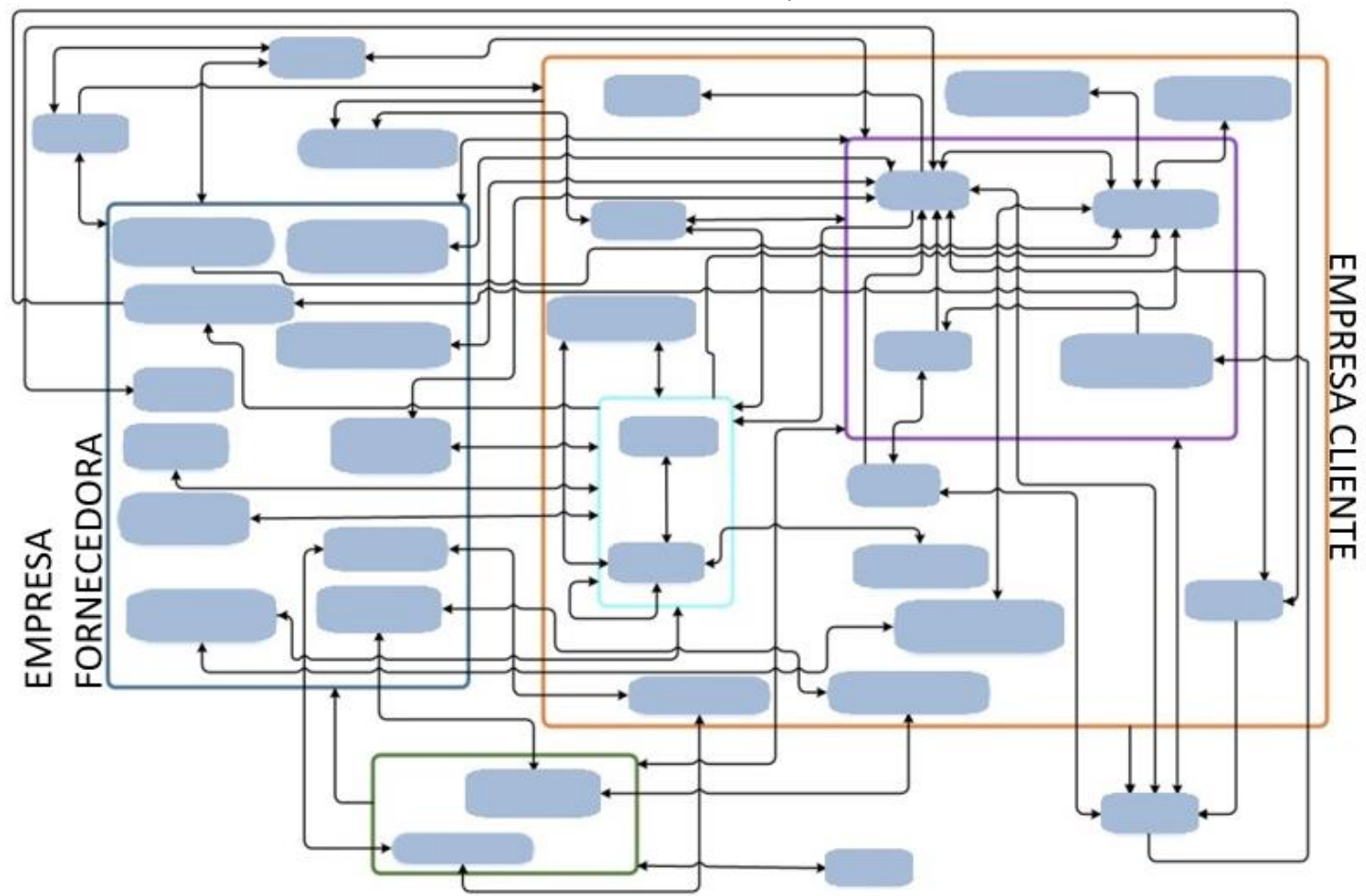

Já o mapa do sistema fornece uma perspectiva mais detalhada sobre o tipo e qual informação é transferida entre atores envolvidos em um SPS. A informação é geralmente dividida em dois tipos, informações de primeiro e segundo nível. Informações de primeiro nível estão diretamente relacionadas ao SPS e à capacidade de fornecê-lo. A informação de segundo nível está indiretamente relacionada ao SPS e é focada no desenvolvimento de potenciais futuras ofertas relacionadas ao SPS (LINDAHL; SAKAO; CARLSSON, 2014).

O mapa do sistema deste estudo de caso é apresentado na figura 2 e fornece uma perspectiva mais detalhada do SPS, mostrando qual informação é trocada entre os atores, identificada pelos números, bem como o fluxo de informação. Como os dados são confidencias, as informações não serão detalhadas neste estudo.

Os mapas são importantes para definir quem são os atores envolvidos, as principais atividades que realizam e como é o fluxo de informação entre eles. Quando se faz esse mapa, é possível comparar como cada um dos entrevistados enxerga o SPS, ou seja, qual o seu nível de conhecimento sobre os atores envolvidos no SPS (LINDAHL; SAKAO; CARLSSON, 2014). Por exemplo, alguns entrevistados identificaram alguns atores mas não sabiam quais atividades desempenhavam e nem como eles interagiam entre si. Portanto, a construção desses mapas é fundamental para que os entrevistados também tenham conhecimento sobre as atividades que cada ator envolvido no desenvolvimento da oferta de SPS desempenha bem como os seus requisitos. Quando não se tem o conhecimento sobre isso, o fluxo de informação pode ser prejudicado, ou seja, pode haver o atraso na troca de informação, afetando o trabalho do grupo como um todo. 
Figura 2. Mapa do sistema do SPS que ilustra as transferências de informações entre os atores. (Mapa alterado devido a confidencialidade).

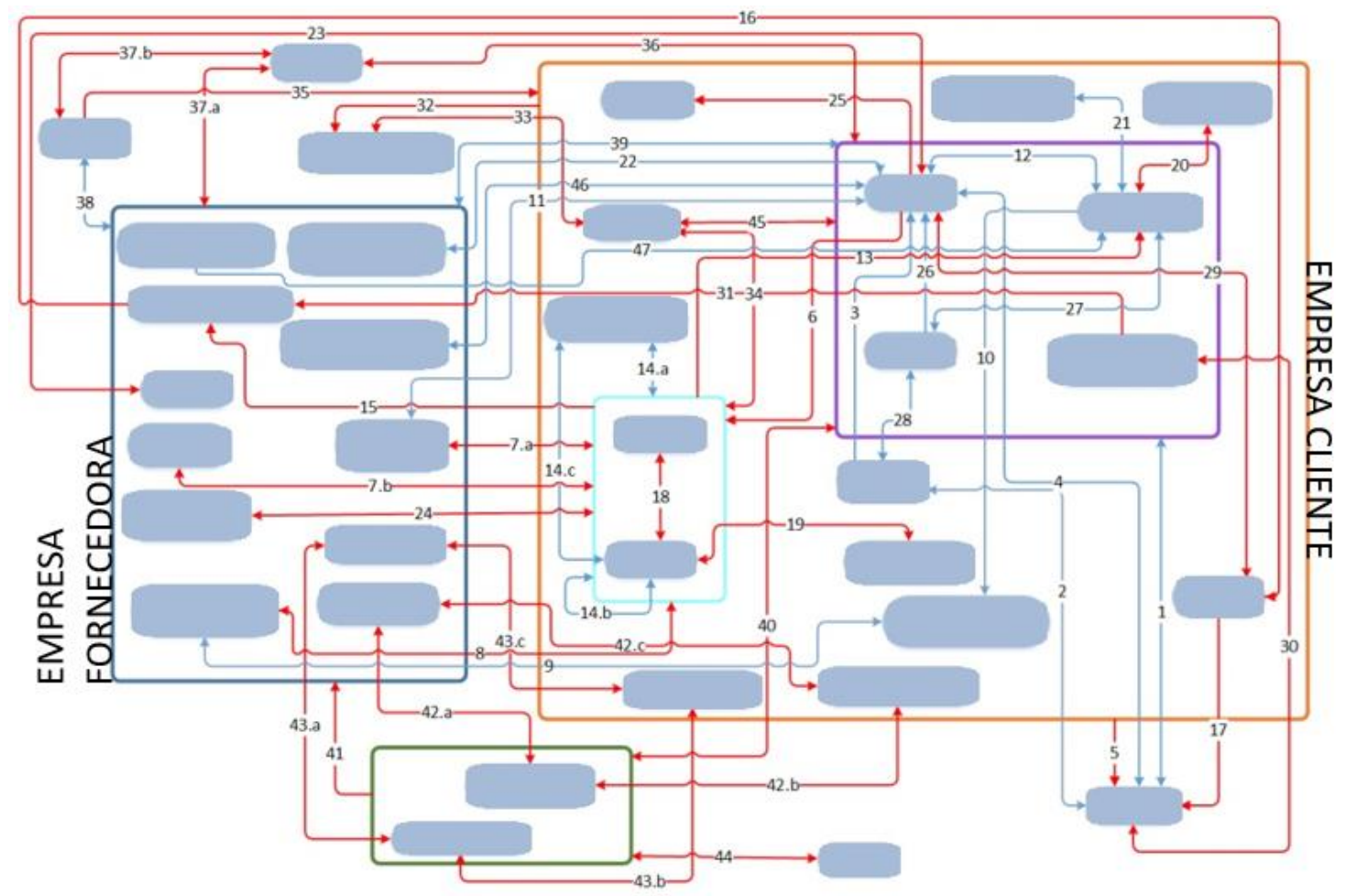

\subsection{Oportunidades de melhorias}

São apresentadas as melhorias necessárias para o SPS ser mais eficiente, identificadas pelos entrevistados da empresa cliente. Essas melhorias podem ser divididas em melhorias na relação cliente-fornecedor e melhorias internas à organização.

\subsubsection{Melhorias na relação cliente-fornecedor}

Com base nas entrevistas, pode-se notar que a principal maneira de melhorar a colaboração entre a empresa cliente e a fornecedora é melhorando a comunicação entre os atores das duas empresas. De acordo com a empresa cliente, os atores da fornecedora muitas vezes são lentos em responder às suas demandas e dúvidas referente a oferta de SPS. Portanto, para a empresa cliente, a fornecedora poderia ser mais eficiente e ágil em termos de resposta. Isso poderia ser feito se as duas empresas tivessem um planejamento comum com prazos a serem seguidos, o que forçaria a empresa fornecedora a não adiar as respostas. Esse planejamento também permitiria que as informações estivessem em sincronia, assim mitigando o risco de atrasar o lançamento da plataforma no mercado.

Alguns entrevistados afirmam que é necessário uma melhoria na sua visão de longo prazo sobre os desenvolvimentos futuros que a fornecedora fará na plataforma. Isso será possível a partir da criação do roadmap pela empresa fornecedora. Para essa melhoria, os entrevistados ressaltam que as duas empresas precisam ter reuniões regulares permitindo-lhes trocar informações importantes sobre o desenvolvimento do SPS.

Alguns entrevistados acreditam que a existência de problemas na comunicação entre as duas empresas se deve ao fato do desenvolvimento do SPS ainda não estar completamente maduro. Assim, por exemplo, a empresa cliente às vezes deseja informações sobre a oferta de SPS, a qual o fornecedor ainda não possui, visto que o SPS ainda está em desenvolvimento. Portanto, é importante que haja uma sincronia entre as informações que cada empresa possui. 


\subsubsection{Melhorias internas à empresa cliente}

Segundo os entrevistados, a empresa deve melhorar seus fluxos internos de informação, pois o principal problema da empresa é a sua lentidão em termos de decisão e execução. Isso gera impactos na transferência de informações entre os atores da própria empresa, pois quando a informação não é repassada para o ator que precisa recebê-la, o tempo de decisão deste acaba sendo prejudicado. Assim, uma reatividade rápida é importante, a qual pode ser obtida por meio do fortalecimento da interação e colaboração entre os departamentos.

\subsection{Pontos fracos e benefícios na compra do SPS}

Durante as entrevistas os respondentes foram questionados sobre os pontos-fracos e os benefícios que enxergavam sobre a compra dessa oferta de SPS. Uma lista extensa sobre os pontos-fracos foi identificada, enquanto poucos benefícios foram listados. Isso se deve ao fato do desenvolvimento do SPS ainda estar no início e da empresa cliente não ter uma visão abrangente sobre os benefícios potenciais do SPS, visto que ainda existem muitas incertezas sobre o mesmo.

\subsubsection{Pontos fracos}

- Perda da propriedade: a empresa cliente se mostra relutante em aceitar que a plataforma não é sua propriedade, sempre questionando quem é o proprietário da mesma. Como as duas empresas compartilham conhecimentos e trocam muitas informações, por exemplo, sobre as evoluções técnicas da plataforma, apesar da empresa cliente não estar desenvolvendo a parte operacional da plataforma, ela questiona sua propriedade. Portanto, para se proteger em termos de propriedade intelectual, a empresa cliente tem elaborado documentos formais para a propriedade intelectual e a confiabilidade da informação;

- Perda do know-how: a empresa cliente afirma que não sabe como a plataforma funciona tecnicamente, uma vez que a empresa compra apenas o serviço. Portanto, ela considera que está perdendo know-how, visto que o fornecedor está operando uma parte que a empresa poderia desenvolver. Isso pode resultar em uma perda do seu próprio knowhow e de habilidades específicas que foram acumuladas ao longo do tempo, já que a empresa não está desenvolvendo esses novos conhecimentos;

- Dependência do fornecedor: a empresa se torna dependente do fornecedor para atender aos seus clientes finais. Uma vez que a empresa fornecedora decida não trabalhar mais em colaboração ou por algum motivo decida não operar mais a plataforma, a empresa cliente será afetada. Portando a empresa cliente se torna dependente do fornecedor assumindo todos os riscos referentes a compra dessa oferta de SPS;

- Perda do poder de decisão: a empresa cliente não pode tomar qualquer decisão referente ao SPS sem consultar a empresa fornecedora, assim os entrevistados afirmam que, comprando a oferta de SPS, perderam seu poder de decisão. A empresa cliente afirma também que não pode tomar decisões apenas com o fornecedor já que as mesmas devem ser acordadas com as outras empresas parceiras;

- Alto risco financeiro: o investimento deste colaboração com a empresa fornecedora é muito alto. A empresa cliente ressalta que a escolha de comprar um SPS traz um alto risco financeiro, pois não possui garantia concreta que essa escolha trará o retorno financeiro esperado quando lançado no mercado, visto que ela não tem o controle total do SPS. 


\subsubsection{Benefícios na compra do SPS}

- Atendimento de novos clientes, ampliação do mercado: a empresa cliente afirma que a compra do SPS permite que ela atenda às necessidades de seus novos clientes internacionais, que ela não conseguiria atender sozinha por não possuir a plataforma adequada para tal;

- Concentração em suas atividades principais: como a empresa cliente não precisa desenvolver tecnicamente a plataforma, ela possui mais tempo e recurso para se concentrar nas atividades principais realizadas atualmente. Portanto, ao comprar o SPS do fornecedor, a empresa cliente é capaz de liberar recursos internos próprios e concentrar-se melhor em suas competências e processos centrais.

\section{DISCUSSÃO}

Há uma série de vantagens ao comprar uma oferta de SPS. De acordo com Rackham e DeVincentis (1999) o cliente deseja uma parceria em que ambas as partes colaboram e desenvolvam o SPS. Ao fazer isso, aumentaria a oportunidade para ambos de entrar conjuntamente em novos mercados e de fornecer novas experiências aos clientes finais. Como foi abordado, um dos benefícios da compra do SPS pela empresa cliente é atender as necessidades de seus novos clientes. Tukker (2004) descreve essa colaboração como uma forma que permite que as empresas melhorem sua posição competitiva no mercado.

Tukker (2004) ressalta a importância de que todos os envolvidos trabalhem para o mesmo objetivo. Portanto, a empresa cliente e a fornecedora ganham de ambos os lados, uma vez que o valor é compartilhado e ambos devem se esforçar para obter conjuntamente mais valor. Assim, uma das principais vantagens dessa colaboração é que mesmo se o provedor e o cliente precisem compartilhar mais informações, eles vão ganhar mais ao mesmo tempo.

As empresas muitas vezes têm seus próprios sistemas de logística, diferentes sistemas de informação ou uma cultura organizacional diferente, mostrando a existência de incertezas na colaboração (SAKAO; RÖNNBÄCK; SANDSTRÖM, 2013). Entrevistados da empresa cliente afirmaram que não estão acostumados a não saber como a parte técnica de uma plataforma funciona, uma vez que eles estão acostumados a desenvolver tecnicamente outras plataformas. Por um lado eles não têm de operar a plataforma fornecida, mas por outro lado eles se sentem desconfortáveis por não ter uma visão completa de como a plataforma de fato funciona. Tukker (2004) descreve isso como sendo um benefício para o cliente, pois isso permite que ele se concentre em suas atividades principais, o qual foi descrito como benefício pela empresa cliente. Tukker (2004) também descreve o SPS como uma boa maneira de ter uma relação de longo prazo entre duas empresas, portanto, é importante que a empresa fornecedora conheça os sentimentos de desconforto da empresa cliente em não saber muito sobre o conteúdo técnico e também que as empresas possuam um planejamento em comum para terem uma visão a longo prazo mais bem definida.

Como abordado por Baines et al (2007) a adoção de uma estratégia de SPS traz desafios culturais e corporativos significativos para as empresas envolvidas. Alguns autores (GOEDKOOP et al., 1999; MONT, 2002) veem a mudança cultural como sendo o principal obstáculo à adoção de um SPS, pois é necessário que o consumidor valorize o atendimento da sua necessidade por meio do serviço ao invés da compra de um produto. Como discutido anteriormente, a empresa cliente é resistente em aceitar que ela não é proprietária da plataforma e assim não conhecerá profundamente como a mesma funciona, portanto uma mudança cultural na empresa cliente é necessária. 
A informação desempenha um papel importante em um SPS, uma vez que traz benefícios e riscos. As razões para que existam riscos são, por exemplo, a falta de informações necessárias por parte de um ator devido à posse de informação de outro ator; ou, simplesmente a inexistência de informação (LINGEGÅRD; SAKAO; LINDAHL, 2012). Como abordado anteriormente, a empresa cliente também precisa mudar sua maneira de trocar informações com a empresa fornecedora e vice-versa. Esta mudança só será possível através da melhoria no fluxo de informação existente entre os atores das duas empresas. Durante as entrevistas, uma das principais reclamações da empresa cliente é a lentidão da resposta por parte do fornecedor, isso mostra claramente que se os atores da empresa cliente não possuem as informações necessárias no momento certo, pode haver um risco na melhoria da colaboração cliente-fornecedor e consequentemente no desenvolvimento do SPS.

\section{CONSIDERAÇÕES FINAIS}

Este trabalho apresentou o estudo de caso de uma empresa francesa de telecomunicação que compra uma oferta SPS, o qual é a plataforma de conexão de dispositivos M2M fornecido como serviço por uma empresa sueca de telecomunicação. Essas duas empresas trabalham em colaboração para desenvolver e aprimorar características técnicas desta plataforma. Como foi observado, para que esta oferta de SPS possa ser melhorado, os atores do cliente e do fornecedor devem ter acesso a informações importantes, saber como elas são transferidas e quem usará as informações que possuem. Portanto, o trabalho identificou e analisou, por meio do mapa dos atores e do mapa do sistema, como os atores da empresa cliente interagem entre si e com outros atores, principalmente com os da empresa fornecedora.

Também foram identificadas melhorias necessárias para que o SPS seja mais eficiente a partir da perspectiva da empresa cliente. Pode-se perceber que a principal maneira de aprimorar a colaboração cliente-fornecedor é melhorando a comunicação entre os atores das duas empresas, por exemplo, por meio de reuniões regulares, de um planejamento comum às duas empresas com prazos definidos e a identificação de atores chaves, por parte das duas empresas, permitindo um fluxo de informação melhor. As melhorias internas também são necessárias, tais como aumentar a rapidez na tomada de decisão, melhorar seu fluxo interno de informações e reforçar a interação entre os departamentos.

Do ponto de vista da empresa cliente, comprar uma oferta de SPS pode trazer benefícios como: atendimento de clientes internacionais e a concentração em suas atividades principais; e também pontos-fracos como: perda do know-how, dependência do fornecedor e perda do poder de decisão.

\section{REFERÊNCIAS}

AHLSTROM, P. Lean service operations: translating lean production principles to service operations. International Journal of Services Technology and Management, v.5, n.5/6, p.545564, 2004.

BABBIE, E. R. The practice of social research. [s.l.] Wadsworth Cengage Learning, 2013.

BAINES, T. S. et al. State-of-the-art in product-service systems. Proceedings of the Institution of Mechanical Engineers, Part B: Journal of Engineering Manufacture, v.221, n.10, p.1543-1552, 2007.

BRINKMANN, S.; KVALE, S. InterViews: Learning the Craft of Qualitative Research Interviewing. 3rd edition ed. s.l.:s.n, 2015. 
GOEDKOOP, M. J. et al. Product Service systems, Ecological and Economic Basics. Amersfoort: Report for Dutch Ministries of Environment (VROM) and Economic Affairs (EZ), 1999.

LINDAHL, M.; SAKAO, T.; CARLSSON, E. Actor's and System Maps for Integrated Product Service Offerings - Practical Experience from Two Companies. Procedia CIRP, v.16, p.320-325, 2014.

LINDAHL, M.; SAKAO, T.; RÖNNBÄCK, A. O. Business Implications of Integrated Product and Service Offerings. CIRP IPS2 Conference 2009, Cranfield, UK, p.165-172, 2009.

LINGEGÅRD, S.; SAKAO, T.; LINDAHL, M. Integrated Product Service Engineering Factors influencing environmental performance. In: Design for Innovative Value Towards a Sustainable Society, p.386-391, 2012.

MONT, O. Clarifying the concept of product-service system. Journal of Cleaner Production, v.10, p.237-245, 2002.

RACKHAM, N.; DEVINCENTIS, J. R. Rethinking the sales force: redefining selling to create and capture customer value. [s.l.] McGraw-Hill, 1999.

SAKAO, T.; RÖNNBÄCK, A. O.; SANDSTRÖM, G. O. Uncovering benefits and risks of integrated product service offerings - Using a case of technology encapsulation. Journal of Systems Science and Systems Engineering, v.22, n.4, p.421-439, 2013.

TUKKER, A. Eight types of product-service system: eight ways to sustainability? Experiences from SusProNet. Business Strategy and the Environment, v.260, n.13, p.246260, 2004.

TUKKER, A.; TISCHNER, U. Product-services as a research field: past, present and future. Reflections from a decade of research. Journal of Cleaner Production, v.14, n.17, p.15521556, 2006. 